\title{
The model of coronal hole with microwave observational data taking into account the solar wind flows
}

\author{
V.N. Borovik ${ }^{1}$, T.I. Kaltman ${ }^{2}$ and A.N. Korzhavin ${ }^{2}$ \\ ${ }^{1}$ Central Astronomical observatory, St.-Petersburg, Russia \\ ${ }^{2}$ Special Astrophysical observatory, St.-Petersburg, Russia email: kti@saoran.spb.su
}

\begin{abstract}
The renew non-hydrostatic model of electron density, temperature and velocity distribution with height in coronal hole $(\mathrm{CH})$ is build. The presented model is based on the measurements of magnetic field in CH with RATAN-600 observations and on the assumption about equality of magnetic and kinetic energy of upward plasma flows in $\mathrm{CH}$ at heights of generation of radio emission in wavelength range $20-30 \mathrm{~cm}$. To construct this model the observational spectrum of brightness temperatures of $\mathrm{CH}$ in wavelength range $2-30 \mathrm{~cm}$ is used. The first results of modeling are presented.
\end{abstract}

\section{Introduction to the model}

The model of Lantos 1972 for interspicular regions was used to determine three functions of altitude: the temperature, the electron density and the expansion velocity. There are four differential equations to be solved: energy and momentum conservation, continuity and an equation deduced from central brightness spectrum at centimetric wavelengths. One of this is rejected as only three functions are to be determined, but it is shown that it is compatible with the others. In addition we must know two boundary conditions in order to obtain the model, as only the relative altitudes are needed. The two parameters to be determined are the electron density $N_{R}$ and the expansion velocity $V_{R}$ for a given electron temperature $T_{R}$ taken as reference. The hypothesis of a plane-parallel atmosphere was made, with uniform and radial magnetic field and with neglecting of mechanical wave dissipation.

(a) Energy conservation equation: $\left(\frac{d J_{q}}{d x}\right)=\left(\frac{d F_{T}}{d x}\right)+E_{\text {rad }}$, where $d J_{q}=K T^{\frac{5}{2}}\left(\frac{d T}{d x}\right)$ the absolute value of the conductive flux, $F_{T}$ - flux of energy transported by the gas in upward movement, $E_{\text {rad }}$ - radiated energy, can be written as:

$$
\left(\frac{d}{d x}\right)\left(K T^{\frac{5}{2}}\left(\frac{d T}{d x}\right)\right)-E_{\text {rad }}-\left(\frac{d}{d x}\right)(5 N v k T)=0,
$$

where $K=10^{-6}$ - the conductivity constant, $E_{\text {rad }}=1.75 \times 10^{2} N^{2} \mathrm{erg} / \mathrm{cm}^{3} / \mathrm{s}$.

(b) Momentum conservation:

$$
N m_{H} v\left(\frac{d v}{d x}\right)+\left(\frac{d}{d x}\right)(2 N k T)=0,
$$

$v$ - the upward velocity of the gas.

(c) Mass continuity:

$$
\left(\frac{d(N V)}{d x}\right)=0
$$

(d) Equation deduced from the radio observations.

The solution of the transfer equation: $T_{b}(\nu)=\int_{0}^{\infty} T\left(\tau_{\nu}\right) e^{-\tau_{\nu}} d \tau_{\nu}$ can be writing as a 
Laplace transform to be inverted and to yield an analytic expression for the electronic temperature (Piddington, 1954).

$$
T_{b}(\nu)=a+b \nu^{-1}+c \nu^{-2}
$$

Inserting this expression for $T_{b}$ and writing the absorption coefficient in the transfer equation, after some change of variables we get the following equation for density:

$$
N^{2}=\left(\frac{2 b^{2}}{\beta \pi}\right) \frac{T^{3 / 2}}{(T-a)^{3}} \frac{d T}{d x}
$$

\section{Results of modeling}

First, we recalculated the Lantos model to construct a method for atmosphere modeling. Then, we applied this method for modern RATAN-600 observational data of free-spot areas and that of coronal holes (Borovik et al. 1999a, Borovik et al. 1999b). Lantos had used the observational data of Piddington 1954.

Solving the system of four differential equation (1)-(4b) we determine pressure, conductive flux, density, velocity as a function of electron temperature and relative altitudes. As the velocity can be obtained from two independent equation (2) or (3), the accordance between these two solutions for velocities confirms (or not) the consistency of four equations considered here. The first boundary condition $V_{R}$ was evaluated from this comparison. The second boundary condition $N_{R}$ was determined from the comparison between theoretical and the observed spectra of brightness temperatures.

We have successfully calculated the Lantos model for spot-free Sun regions for small velocities. But it was not so satisfactory to construct a coronal hole model with high velocities as the method used here was not enough stable at such conditions. Our advanced model for $\mathrm{CH}$ is based on the measurements of magnetic field in $\mathrm{CH}$ with RATAN-600 observations (Borovik et al. 1999b) and on the assumption about equality of magnetic and kinetic energy of flows in $\mathrm{CH}$ at heights of generation of radio emission in wavelength range $20-30 \mathrm{~cm}$. To construct this model the observational spectrum of brightness temperatures of $\mathrm{CH}$ in wavelength range $2-30 \mathrm{~cm}$ was used. It was assumed that the matter flows in $\mathrm{CH}$ directed up with velocities $\mathrm{v}=0$ at the photosphere level and $v=500-1000$ $\mathrm{km} \mathrm{c}^{-1}$ at heights of generation of radio emission of $\mathrm{CH}$ in wavelength range $20-30 \mathrm{~cm}$. As we were waiting for, we received the densities smaller then that of quiet Sun regions, and the smaller gradient of densities and temperature distributions. The first results of modeling presented here allow us to more investigate this method and to improve it to receive the distributions of physical parameters for coronal holes according to modern observations.

\section{Acknowledgements}

This paper is supported by Program OFN-18 of RAS, by Dogovor N $16 \mathrm{~K}$ and by RBRF under grants number 03-02-17357, 03-02-17528.

\section{References}

Lantos P. 1972 Solar physics 22, 387-401

Piddington J.H. 1954 ApJ 119, 531-540

Borovik V.N. \& Medar V.G. 1999 proceedings 8-th SOHO Workshop, Paris, France, 22-25 June 1999, (ESA SP-446, October 1999), 185-189

Borovik V.N., Medar V.G. \& Korzhavin A.N., 1999 Pisma v Astronomicheskii Zhurnal, 1999, 25, 4, 299-307 